\title{
LRS Bianchi Type-I Inflationary String Cosmological Model in Brans-Dicke Theory of Gravitation
}

\author{
R. Venkateswarlu ${ }^{1}$ and J. Satish ${ }^{2}$ \\ ${ }^{1}$ GITAM School of Int'l Business, GITAM University, Visakhapatnam 530045, India \\ ${ }^{2}$ Vignan's Institute of Engineering for Women, Visakhapatnam 530046, India \\ Correspondence should be addressed to J. Satish; satish_janjeti@yahoo.co.in
}

Received 14 May 2014; Revised 13 July 2014; Accepted 18 July 2014; Published 20 August 2014

Academic Editor: Kazuharu Bamba

Copyright (C) 2014 R. Venkateswarlu and J. Satish. This is an open access article distributed under the Creative Commons Attribution License, which permits unrestricted use, distribution, and reproduction in any medium, provided the original work is properly cited.

\begin{abstract}
We investigate locally rotational symmetric (LRS) Bianchi type I space time coupled with scalar field. String cosmological models generated by a cloud of strings with particles attached to them are studied in the Brans-Dicke theory. We assume that the expansion scalar is proportional to the shear scalar and also power law ansatz for scalar field. The physical behavior of the resulting model is discussed through different parameters.
\end{abstract}

\section{Introduction}

It is believed that the early universe evolved through some phase transitions, thereby yielding a vacuum energy density which at present is at least 118 orders of magnitudes smaller than in the Planck time [1]. Such a discrepancy between theoretical expectations and empirical observations constitutes a fundamental problem in the interface uniting astrophysics, particle physics, and cosmology. The recent observational evidence for an accelerated state of the present universe, obtained from distant SNe Ia (Perlmutter et al. [2]; Riess et al. [3]), gave strong support to search for alternative cosmologies. Thus, the state of affairs has stimulated the interest in more general models containing an extra component describing dark energy and simultaneously accounting for the present accelerated stage of the universe.

The isotropic models are considered to be the most suitable to study large scale structure of the universe. However, it is believed that the early universe may not have been exactly uniform. This prediction motivates us to describe the early stages of the universe with the models having anisotropic background. Thus, it would be worthwhile to explore anisotropic models in the context of modified theories of gravity. Among the various modifications of general relativity (GR), the Brans-Dicke (BD) theory of gravity [4] is a well-known example of a scalar tensor theory in which the gravitational interaction involves a scalar field and the metric tensor. One extra parameter $\omega$ is used in this theory which satisfies the equation given by

$$
\square \phi=\frac{8 \pi T}{\phi(3+2 \omega)},
$$

where $\phi$ is known as BD scalar field while $T$ is the trace of the matter energy-momentum tensor. It is mentioned here that the general relativity is recovered in the limiting case $\omega \rightarrow$ $\infty$. Thus we can compare our results with experimental tests for significantly large value of $\omega$. The majority of popular cosmological models, including all the ones referred to above, use the cosmological principle; that is, they assume that the universe is homogeneous and isotropic. On the other hand, there are hints in the $\mathrm{CMB}$ temperature anisotropy studies that suggest that the assumption of statistical isotropy is broken on the largest angular scales, leading to some intriguing anomalies. To provide predictions for the CMB anisotropies, one may consider the homogeneous but anisotropic cosmologies known as Bianchi type space times, which include the isotropic and homogeneous FRW models. In this study we consider the simplest of these, the locally rotationally symmetric (LRS) Bianchi type-I space time as an anisotropic background universe model; note that this space time is a generalization of flat $(k=0)$ FRW metric. 
The presence of strings results in anisotropy in the space time, though strings are not observable in the present epoch. Unlike domain walls and monopoles, strings cause no harm (to the cosmological models) but rather can lead to very interesting astrophysical consequences. The string gas cosmology will lead to a dynamical evolution of the early universe, very different from what is obtained in standard and inflationary cosmology and can already be seen by combining the basic ingredients from string theory discussed so far. As the radius of a cloud of strings decreases from an initially large value which maintains thermal equilibrium, the temperature first rises as in standard cosmology since the occupied string states (the momentum modes) get heavier. However, as the temperature approaches the Hagedorn temperature, the energy begins to flow into the oscillatory modes and the increase in temperature levels off. As the radius decreases below the string scale, the temperature begins to decrease as the energy begins to flow into the winding modes whose energy decreases as the radius decreases.

Brans-Dicke theory has been studied by several researchers in different contexts. Johri and Desikan [5] obtained Brans-Dicke cosmological models with constant deceleration parameter in the presence of particle creation. Ram and Singh [6] studied the effect of time dependent bulk viscosity on the radiation of Friedmann models with zero curvature in BD theory. Singh and Beesham [7] studied the effect of bulk viscosity on the evolution of the spatially flat FriedmannLemaitre-Robertson-Walker models in the context of open thermodynamical systems, which allow for particle creation, analyzed within the framework of BD theory. Reddy et al. [8] discussed the homogeneous axially symmetric Bianchi type-I radiating cosmological model with negative constant deceleration parameter in BD scalar-tensor theory gravitation. Spatially homogeneous and anisotropic Bianchi type-I (BI) model is used to study the possible effects of anisotropy in the early universe $[9,10]$. Some people [11-13] have constructed cosmological models by using anisotropic fluid and BI universe. Recently, this model has been studied in the presence of binary mixture of the perfect fluid and the DE [14]. Sharif and Kausar $[15,16]$ have discussed dynamics of the universe with anisotropic fluid and Bianchi models in $f(R)$ gravity. Some exact BI solutions have also been investigated in this modified theory [17-20].

In this paper, we consider a spatially homogeneous and anisotropic LRS Bianchi type-I cosmological model in BransDicke scalar-tensor theory of gravitation in the presence of cosmic string source. Some physical properties of the model are also discussed. The resulting cosmological model can be considered as an analogue of inflationary model in BransDicke string cosmology.

\section{Metric and Field Equations}

The line element for the spatially homogeneous and anisotropic LRS Bianchi type-I space time is given by

$$
d s^{2}=-d t^{2}+A^{2} d x^{2}+B^{2}\left(d y^{2}+d z^{2}\right)
$$

where $A$ and $B$ are functions of $t$ only. Brans-Dicke theory of gravitation is a natural extension of general relativity which introduces an additional scalar field $\phi$ besides the metric tensor $g_{i j}$ and dimensionless coupling constant $\omega$. The scalar tensor field equations in the Brans-Dicke theory are given by the field equation

$$
\begin{aligned}
R_{i j}-\frac{1}{2} g_{i j} R= & \omega \phi^{-2}\left(\phi_{, i} \phi_{, j}-\frac{1}{2} g_{i j} \phi_{, k} \phi^{, k}\right) \\
& -\phi^{-1}\left(\phi_{i j}-g_{i j} \square \phi\right),
\end{aligned}
$$

where

$$
\square \phi=\phi_{, k}^{, k}=\frac{8 \pi T}{\phi(3+2 \omega)} .
$$

Here $T_{i j}$ is the energy momentum tensor of matter, $\phi$ the scalar field, and $\omega$ the dimensionless coupling constant. As a consequence of field equations (2) and (3) we get the equation

$$
T_{, i}^{i j}=0 .
$$

The energy momentum tensor for a cloud of strings is given by

$$
T_{i j}=\rho u_{i} u_{j}-\lambda x_{i} x_{j}
$$

Here, $\rho$, the proper energy density, and $\lambda$, the string tension density, are related by $\rho=\rho_{p}+\lambda$, where $\rho_{p}$ is the particle density of the configuration. The velocity $u^{i}$ describes the 4 -velocity which has components $(1,0,0,0)$ for a cloud of particles and $x^{i}$ represents the direction of string which will satisfy

$$
\begin{gathered}
u^{i} u_{i}=-x^{i} x_{i}=1, \\
u^{i} x_{i}=0 .
\end{gathered}
$$

The field equations (3) for the metric (2) are given by

$$
\begin{gathered}
2 \frac{\ddot{B}}{B}+\frac{\dot{B}^{2}}{B^{2}}=\frac{8 \pi \lambda}{\varphi}-\frac{\omega}{2}\left(\frac{\dot{\phi}}{\phi}\right)^{2}+\frac{\ddot{\phi}}{\phi}+2 \frac{\dot{\phi} \dot{B}}{\phi B}, \\
\frac{\ddot{A}}{A}+\frac{\ddot{B}}{B}+\frac{\dot{A} \dot{B}}{A B}=-\frac{\omega}{2}\left(\frac{\dot{\phi}}{\phi}\right)^{2}+\frac{\ddot{\phi}}{\phi}+\frac{\dot{\phi}}{\phi}\left(\frac{\dot{A}}{A}+\frac{\dot{B}}{B}\right), \\
\frac{2 \dot{A} \dot{B}}{A B}+\frac{\dot{B}^{2}}{B^{2}}=\frac{8 \pi \rho}{\phi}+\frac{\omega}{2}\left(\frac{\dot{\phi}}{\phi}\right)^{2}+\frac{\dot{\phi}}{\phi}\left(\frac{\dot{A}}{A}+2 \frac{\dot{B}}{B}\right), \\
\ddot{\phi}+\dot{\phi}\left(\frac{\dot{A}}{A}+2 \frac{\dot{B}}{B}\right)=\frac{8 \pi(\rho+\lambda)}{\phi(3+2 \omega)} \\
\dot{\rho}+(\rho-\lambda) \frac{\dot{A}}{A}+\rho \frac{2 \dot{B}}{B}=0 .
\end{gathered}
$$

A “dot" denotes differentiation with respect to $t$. Here we have four independent equations in five unknowns $A, B, \phi$, $\rho$, and $\lambda$. Therefore, we need more relations to find the determinate solutions of these equations. So any one quantity may 
be chosen freely to solve the system of equations. Since the field equations contain $A$ and $B$ and their derivatives, so without any loss of generality, we shall assume that the $\mathrm{BD}$ scalar field $\phi$ is some power of the average scale factor; that is, the power law relation between scale factor a and scalar field $\phi$ has already been used by Johri and Desikan [5] in the context of Robertson Walker Brans-Dicke models.

Thus the power law relation between $\phi$ and scale factor $a$, that is,

$$
\phi \propto a^{\alpha}
$$

where $\alpha$ is any integer, implies that $\phi=b a^{\alpha}$ where $a=$ $\left(A B^{2}\right)^{1 / 3}$ and without loss of generality $b$ can be set equal to 1 .

The corresponding average scale factor $a(t)$, volume $V$, and the mean Hubble parameter $H$ are

$$
\begin{gathered}
a(t)=\left(A B^{2}\right)^{1 / 3}, \\
V=a^{3}(t)=A B^{2}, \\
H=\frac{1}{3}\left(\frac{\dot{A}}{A}+2 \frac{\dot{B}}{B}\right) .
\end{gathered}
$$

The directional Hubble parameters in $x, y$, and $z$ directions are given by

$$
\begin{gathered}
H_{x}=\frac{\dot{A}}{A}, \\
H_{y}=H_{z}=\frac{\dot{B}}{B} .
\end{gathered}
$$

The anisotropy parameter of expansion $\Delta=0$ and the deceleration parameter $q$ are

$$
\begin{gathered}
\Delta=\frac{1}{3} \sum_{i=1}^{3}\left(\frac{H_{i}-H}{H}\right)^{2}, \\
q=\frac{d}{d t}\left(\frac{1}{H}\right)-1 .
\end{gathered}
$$

The isotropic expansion of the universe can be obtained for $\Delta=0$. The expansion and shear scalar turn out to be

$$
\begin{gathered}
\theta=\frac{\dot{A}}{A}+2 \frac{\dot{B}}{B}, \\
\sigma=\frac{1}{\sqrt{3}}\left(\frac{\dot{A}}{A}-\frac{\dot{B}}{B}\right) .
\end{gathered}
$$

\section{Cosmological Solutions}

Since the field equations are highly nonlinear, we assume power law for the scalar field $\phi=a^{\alpha}, \alpha \geq 0$, for the expanding universe. For a spatially homogeneous metric, the normal congruence to homogeneous expansion implies that $\sigma / \theta=n$ where $n$ is constant; that is, "the expansion scalar $\theta$ is proportional to shear scalar $\sigma$." This condition leads to

$$
\frac{1}{\sqrt{3}}\left(\frac{\dot{A}}{A}-\frac{\dot{B}}{B}\right)=n\left(\frac{\dot{A}}{A}+2 \frac{\dot{B}}{B}\right)
$$

which yields to

$$
\frac{\dot{A}}{A}=m \frac{\dot{B}}{B}
$$

where $m=(1+2 n \sqrt{3}) /(1-n \sqrt{3})$ and $n$ are constants. Equation (19), after integration, reduces to

$$
A=\beta B^{m}
$$

where $\beta$ is a constant of integration and assumed that $\beta=1$.

The average scale factor for this model of the universe is $a(t)=\left(A B^{2}\right)^{1 / 3}$ and hence $\phi$ takes the form

$$
\phi=B^{(m+2) \alpha / 3} .
$$

Inserting the value of $\phi$ in (9) and using (20), the metric coefficient $B$ becomes

$$
\frac{\ddot{B}}{B}+\frac{\dot{B}^{2}}{B^{2}}\left(\frac{(m+2)^{2}(\omega-2) \alpha^{2}-6(m+2) \alpha m+18 m^{2}}{18(m+1)-6(m+2) \alpha}\right)=0
$$

which on integration yields to

$$
B=(k+1)^{1 /(k+1)}\left(c_{1} t+c_{2}\right)^{1 /(k+1)}
$$

and from (20) leads to

$$
A=(k+1)^{m /(k+1)}\left(c_{1} t+c_{2}\right)^{m /(k+1)},
$$

where

$$
k=\frac{(m+2)^{2}(\omega-2) \alpha^{2}-6(m+2) \alpha m+18 m^{2}}{18(m+1)-6(m+2) \alpha} .
$$

The scalar field was obtained as

$$
\phi=(k+1)^{(m+2) \alpha / 3(k+1)}\left(c_{1} t+c_{2}\right)^{(m+2) \alpha / 3(k+1)} .
$$

The scalar field $\phi$ is found to be an increasing function of time $t$. At the beginning of the universe the scalar field has a significant role in establishing a string dominated era. At large cosmic time, when the effect of the scalar field is negligible, it is seen that particles dominate over the strings to fill up the volume of the universe.

The directional Hubble parameters $H_{i}$ become

$$
H_{x}=\frac{m c_{1}}{(k+1)\left(c_{1} t+c_{2}\right)} \quad H_{y}=H_{z}=\frac{c_{1}}{(k+1)\left(c_{1} t+c_{2}\right)}
$$

while the mean generalized Hubble parameter becomes

$$
H=\frac{(m+2) c_{1}}{(k+1)\left(c_{1} t+c_{2}\right)} .
$$


The string energy density $\rho$, tension density $\lambda$, and the particle density $\rho_{p}$ are given by

$$
\begin{aligned}
\frac{8 \pi \rho}{\phi}= & {\left[\frac{c_{1}^{2}}{9(k+1)^{2}\left(c_{1} t+c_{2}\right)^{2}}\right] } \\
& \times[((m+1)(9 m+9-k) \\
& \quad-(m+2) \alpha\{(m+2) \alpha-6(m+2)-3 k\})], \\
\frac{8 \pi \lambda}{\phi}= & \frac{c_{1}^{2}(1-m)}{(k+1)^{2}\left(c_{1} t+c_{2}\right)^{2}}(3(1+m)-(m+2) \alpha-k),
\end{aligned}
$$

$$
\begin{aligned}
& \frac{8 \pi \rho_{p}}{\phi} \\
& =\frac{c_{1}^{2}}{(k+1)^{2}\left(c_{1} t+c_{2}\right)^{2}}\left(4 m^{2}+2 m-2\right)-2 k m \\
& \quad-\left[\frac{(m+2)^{2} \alpha^{2}-\left(3 m^{2}-33 m-6\right) \alpha-3 k(m+2) \alpha}{9}\right] .
\end{aligned}
$$

We get geometric strings (or) Nambu strings when $\rho=\lambda$ so we have

$$
\begin{aligned}
\rho=\lambda= & {\left[\frac{c_{1}^{2}(1-m)}{(k+1)^{2}\left(c_{1} t+c_{2}\right)^{2}}\right] } \\
& \times\left[\frac{\left\{\left(m^{3}+5 m^{2}+8 m+4\right) \alpha^{2}\right\}+\left(m^{3}+34 m^{2}+45 m-22\right) \alpha+\left(9 m^{3}-214 m^{2}-447 m+258\right)}{\left(3 m^{2}-m+14\right)}\right]
\end{aligned}
$$

where

$$
k=\frac{\left\{\left(m^{3}+5 m^{2}+8 m+4\right) \alpha^{2}+\left(6 m^{3}+39 m^{2}+57 m+6\right) \alpha-54\left(5 m^{2}+9 m-4\right)\right\}}{\left(3 m^{2}-m+14\right)}
$$

and the volume scale factor turns out to be

$$
V=(k+1)^{(m+2) /(k+1)}\left(c_{1} t+c_{2}\right)^{(m+2) /(k+1)} .
$$

The expansion scalar $\theta$ and shear scalar $\sigma$ take the form

$$
\begin{gathered}
\theta=\frac{(m+2) c_{1}}{(k+1)\left(c_{1} t+c_{2}\right)}, \\
\sigma=\frac{1}{\sqrt{3}} \frac{(m-1) c_{1}}{(k+1)\left(c_{1} t+c_{2}\right)} .
\end{gathered}
$$

The mean anisotropy parameter $A_{m}$ becomes

$$
A_{m}=\frac{(2 m+4)^{2}}{3(m+2)^{2}} \text {. }
$$

Using (20) and (23) and the mean Hubble parameter $H$, we can write the deceleration parameter as

$$
q=-\left[1-\frac{(3 k+1)}{(m+2)}\right]
$$

We note that $q<0, q=0$, and $q>0$, respectively, indicate an accelerated expansion, uniform expansion, and the decelerating phase of the universe. Here the deceleration parameter $q$ is found to be negative, which shows that the model inflates. From the expression of the volume of this model it is seen that this model follows the power law of inflation for $k<(m+1) / 3$. The anisotropy parameters are constant at initial epoch and go to zero for later times. The anisotropy parameter of expansion is zero as $m=-2$, at the initial epoch. Since the anisotropy parameter of expansion is constant (it vanishes for $m=-2)$, therefore the model does not isotropize for later times. In this case, the deceleration parameter $q$ is found to be a dynamical quantity and can be negative for the appropriate values of the constant parameters. The universe exhibits an initial singularity of the POINT type at $t=-c_{2} / c_{1}$. The space time is well behaved in the range $\left(-c_{2} / c_{1}\right)<t<\infty$. For physically realistic models, we take $c_{1}, c_{2}>0$. At the initial moment $t=\left(-c_{2} / c_{1}\right)$ the physical and kinematical parameters $\rho, \lambda, \theta, \sigma^{2}$, and $H$ tend to infinity. So the universe starts from an initial singularity with an infinite energy density with an infinite rate of shear and expansion. Moreover, $\rho, \lambda, \theta, \sigma^{2}$, and $H$ tend to zero at the epoch $t=\infty$. Thus $\rho, \lambda, \theta, \sigma^{2}$, and $H$ are monotonically decreasing toward a zero quantity for $t$ in the range $\left(-c_{2} / c_{1}\right)<t<\infty$. The shear tends to zero much faster than the expansion. The proper volume $V$ and the scalar field $\phi$ tend to zero at the initial singularity. As time proceeds the universe approaches an infinitely large volume and an infinitely large scalar field in the limit as $t \rightarrow \infty$. The expansion scalar $\theta$ is always positive in the interval $\left(-c_{2} / c_{1}\right)<$ $t<\infty$. Therefore, the model describes an expanding cosmological model. The deceleration parameter $q$ is constant in the interval $\left(-c_{2} / c_{1}\right)<t<\infty$. 


\section{Conclusions}

In this paper, we observed that all cosmological models evolve with an initial singularity of the POINT type. The universe starts from an initial singularity with an infinite energy density and with an infinite rate of shear and expansion. The proper volume and the scalar field approach zero at the initial singularity and approach an infinitely large value in the limit as $t \rightarrow \infty$. The models describe expanding cosmological models with a constant deceleration parameter. The entire model is highly anisotropic at the time of the evolution of the universe. At the instant of the beginning of this model, universe the scalar field is found to be a constant quantity; it then increases gradually with time. For very close to the big bang singularity, matter will be in highly dense exotic form that may include viscosity, heat flow, and null radiation flow as well as cosmic strings [9]. It is therefore very important that we have a space-time metric with time-dependent scalar field which is capable of describing almost all these attributes for suitable values for certain parameters. The energy conditions are satisfied. We have found a new solution for inflation that deserves attention.

\section{Conflict of Interests}

The authors declare that there is no conflict of interests regarding the publication of this paper.

\section{References}

[1] S. Weinberg, "The cosmological constant problem," Reviews of Modern Physics, vol. 61, no. 1, pp. 1-23, 1989.

[2] S. Perlmutter, G. Aldering, G. Goldberg et al., "Measurements of $\Omega$ and $\Lambda$ from 42 high-redshift supernovae," The Astrophysical Journal, vol. 517, no. 2, pp. 565-586, 1999.

[3] A. G. Riess, A. V. Filippenko, P. Challis et al., "Observational evidence from supernovae for an accelerating universe and a cosmological constant," The Astronomical Journal, vol. 116, no. 3, pp. 1009-1038, 1998.

[4] C. Brans and R. H. Dicke, "Mach's principle and a relativistic theory of gravitation," Physical Review, vol. 124, pp. 925-935, 1961.

[5] V. B. Johri and K. Desikan, "Cosmological models with constant deceleration parameter in Brans-Dicke theory," General Relativity and Gravitation, vol. 26, no. 12, pp. 1217-1232, 1994.

[6] S. Ram and C. P. Singh, "Early cosmological models with bulk viscosity in Brans-Dicke theory," Astrophysics and Space Science, vol. 254, no. 1, pp. 143-150, 1997.

[7] G. P. Singh and A. Beesham, "Bulk viscosity and particle creation in Brans-Dicke theory," Australian Journal of Physics, vol. 52, no. 6, pp. 1039-1049, 1999.

[8] D. R. K. Reddy, R. L. Naidu, and V. U. M. Rao, "A cosmological model with negative constant deceleration parameter in BransDicke theory," International Journal of Theoretical Physics, vol. 46, no. 6, pp. 1443-1448, 2007.

[9] P. Class, A. S. Jakubi, V. Méndez, and R. Maartens, "Cosmological solutions with nonlinear bulk viscosity," Classical and Quantum Gravity, vol. 14, p. 3363, 1997.
[10] A. Pradhan and P. Pandey, "Some bianchi type I viscous fluid cosmological models with a variable cosmological constant astrophys," Space Science, vol. 301, p. 221, 2006.

[11] D. C. Rodrigues, "Anisotropic cosmological constant and the CMB quadrupole anomaly," Physical Review D, vol. 77, Article ID 023534, 2008.

[12] T. Koivisto and D. F. Mota, "Vector field models of inflation and dark energy," Journal of Cosmology and Astro Particle Physics, vol. 8, pp. 21-45, 2008.

[13] T. Koivisto and D. F. Mota, "Accelerating cosmologies with an anisotropic equation of state," Astrophysical Journal Letters, vol. 679, no. 1, pp. 1-5, 2008.

[14] A. K. Yadav and B. Saha, "LRS Bianchi-I anisotropic cosmological model with dominance of dark energy," Astrophysics and Space Science, vol. 337, pp. 759-765, 2012.

[15] M. Sharif and H. R. Kausar, "Anisotropic fluid and Bianchi type III model in $\mathrm{f}(\mathrm{R})$ gravity," Physics Letters $B$, vol. 697, no. 1, pp. $1-6,2011$

[16] M. Sharif and H. R. Kausar, "Non-vacuum solutions of Bianchi type VI0 universe in $\mathrm{f}(\mathrm{R})$ gravity," Astrophysics and Space Science, vol. 332, no. 2, pp. 463-471, 2011.

[17] D. Lorenz-Petzold, "Exact Brans-Dicke Bianchi type-I solutions with a cosmological constant," Physical Review D, vol. 29, no. 10, pp. 2399-2401, 1984.

[18] D. Lorenz-Petzold, "Electromagnetic Brans-Dicke-Bianchi type-V solutions," Astrophysics and Space Science, vol. 114, no. 2, pp. 277-284, 1985.

[19] S. Kumar and C. P. Singh, "Exact bianchi type-I cosmological models in a scalar-tensor theory," International Journal of Theoretical Physics, vol. 47, no. 6, pp. 1722-1730, 2008.

[20] S. Lee, "Anisotropic universe with anisotropic matter," Modern Physics Letters A, vol. 26, p. 2159, 2011. 

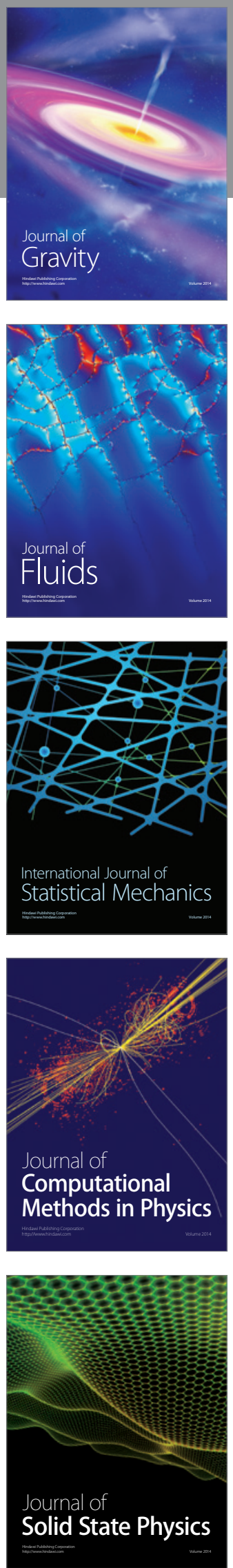

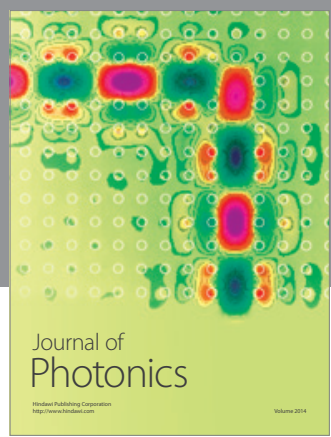

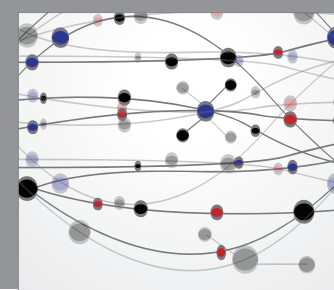

The Scientific World Journal

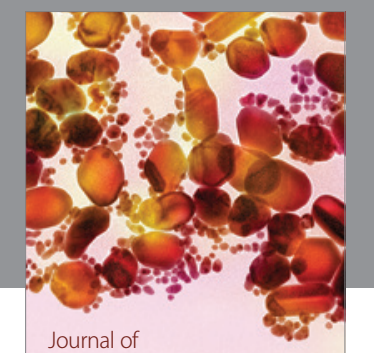

Soft Matter
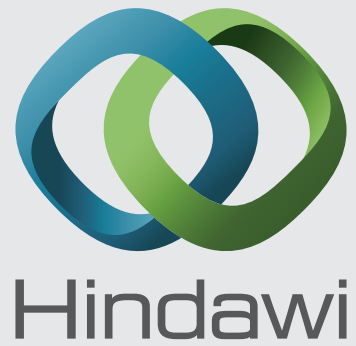

Submit your manuscripts at

http://www.hindawi.com
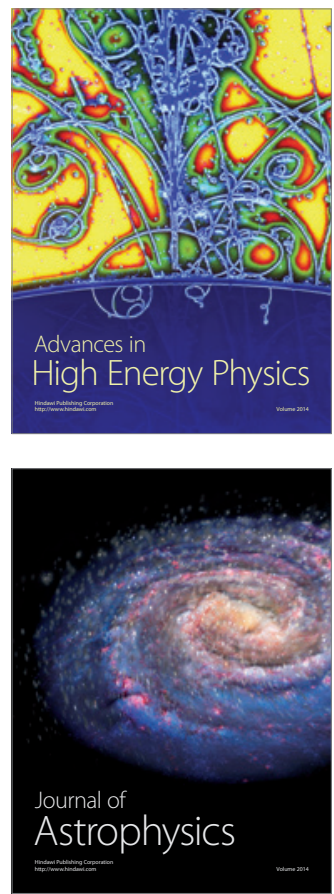
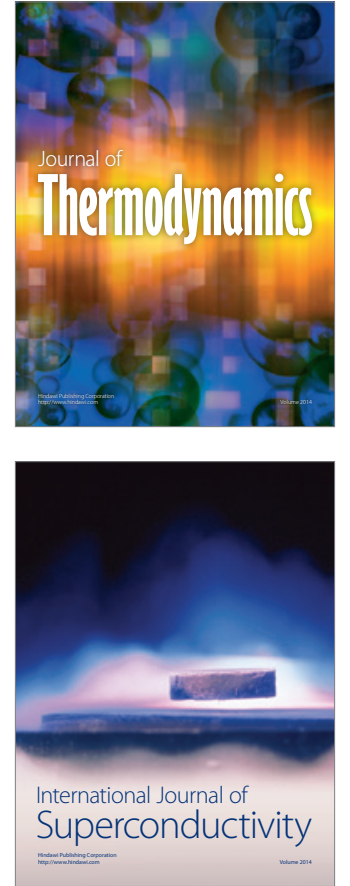
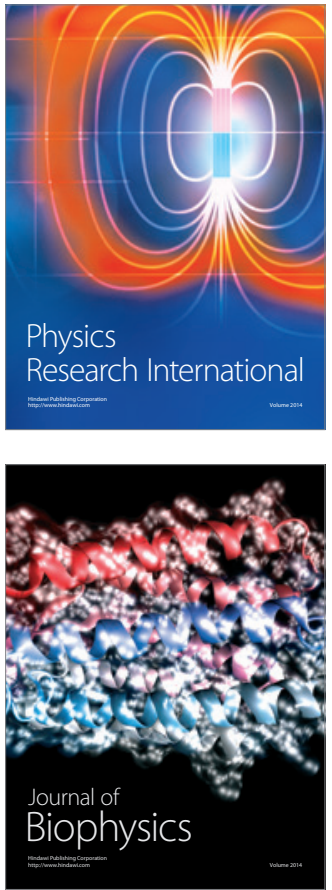
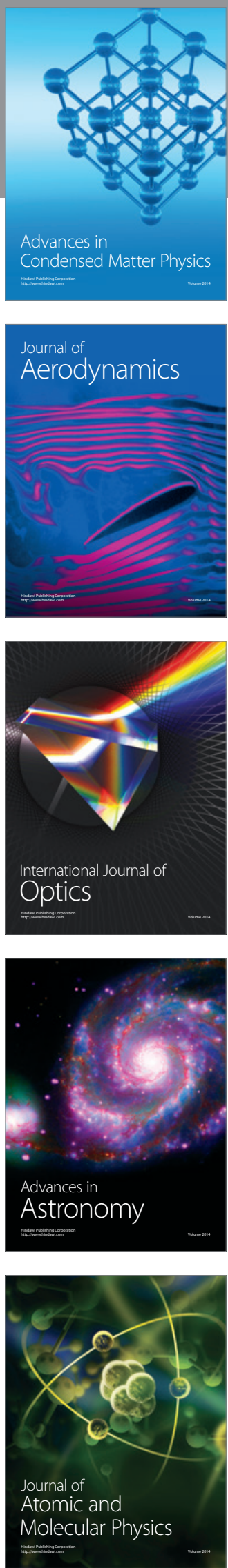